\title{
Solving the Structures of Polycrystalline Materials: from the Debye-Scherrer Camera to SwissFEL
}

\author{
Lynne B. McCusker* and Christian Baerlocher
}

\begin{abstract}
The development of powder diffraction (PD) techniques for structure analysis is traced from its inception almost 100 years ago to the present day, with a brief glimpse of what SwissFEL can contribute in the near future. Although PD data were used in the early days to deduce some simple high-symmetry structures, it was not until computers, instrumentation and synchrotrons arrived on the scene that the true potential of PD data could be realized. In the last 25 years, PD has blossomed into a viable method, not only for structure refinement, but also for structure solution. This means that scientists with polycrystalline materials that cannot be grown as single crystals can still obtain the structural information they need. Historically, structure solution from PD data began with model building, progressed through the application of single-crystal methods to simpler structures and the adaptation of those methods to the special problems posed by PD data, the development of automated model-building algorithms, and most recently to the application of charge flipping. As X-ray sources and detectors continue to develop, the boundary between a powder and a single crystal is narrowing. Laue microdiffraction techniques and the prospects offered by SwissFEL will allow single-crystal data to be collected on some polycrystalline materials.
\end{abstract}

Keywords: Crystal structure analysis · Crystallography · Polycrystalline materials · Powder diffraction · Synchrotron radiation

\section{Introduction}

Structural information is fundamental to the understanding of the properties of any material, whether it be of biological, geological or chemical origin, so it is important to know what tools are available to characterize a material's structure. In the case of crystalline materials, which cover a large fraction of all materials, singlecrystal X-ray diffraction is generally considered to be the method of choice. The structure analysis algorithms that have been developed over the last 100 years of modern crystallography are remarkably robust and yield the structural detail required in over $90 \%$ of the cases. However, even though reliable single-crystal data can now be collected on crystals as small as a few microns in diameter, there are still many materials that are crystalline but do not contain crystals suitable for single-crystal

\footnotetext{
${ }^{\star}$ Correspondence: Dr. L. B. McCusker Laboratory of Crystallography ETH Zurich

Vladimir-Prelog-Weg 5

$\mathrm{CH}-8093$ Zurich

Tel.: +4144632 3721

E-mail: mccusker@mat.ethz.ch
}

analysis. In this case, the use of X-ray powder diffraction (XPD) techniques is often the only option.

XPD is generally viewed as being the poor cousin of its single-crystal counterpart, but an XPD pattern actually contains a surprising amount of information. In principle, the intensities of the reflections measured in a powder diffraction pattern are identical to those measured in a singlecrystal experiment. The problem is that the large number of randomly oriented crystallites that are irradiated in a powder experiment ( $\mathrm{ca} .10^{6}$ crystallites) yield the same number of randomly oriented threedimensional (3D) single-crystal diffraction patterns, all superimposed upon one another. As a result, reflections that have similar $d$-spacings will be recorded at the same time, because they have similar diffraction angles. That is, only the sum of their intensities can be measured. This is known as the reflection overlap problem, and is the major hindrance to structure analysis using powder diffraction data (Fig. 1).

Nonetheless, with the excellent instrumentation, X-ray sources and computing power that we currently have at our disposal, it is now possible to determine crystal structures from such data. In the following sections, we will take a historical view, from a chemist's perspective, of the development of the field since the first X-ray powder diffraction experiments were performed almost 100 years ago. The

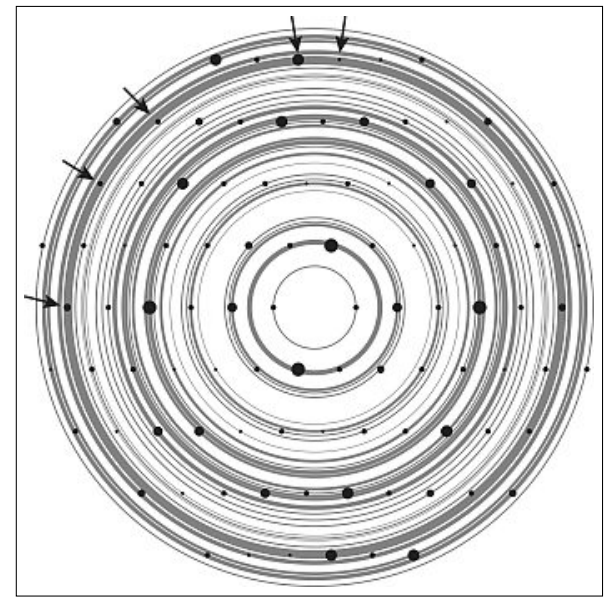

Fig. 1. A 2D single-crystal diffraction pattern overlaid on the corresponding powder diffraction pattern. One group of overlapping reflections is indicated with arrows. The diffraction angle $2 \theta$ increases radially from the center of the diffraction pattern.

possibilities for polycrystalline materials that are opening up in the area of X-ray microdiffraction will also be touched upon.

For more detailed discussions of XPD methodology, the reader is referred to the book entitled 'Structure determination from Powder Diffraction Data' published in $2002,{ }^{[1]}$ the special issue of Zeitschrift für Kristallographie devoted to the same topic published in 2004,[2] and the new volume $\mathrm{H}$ of the International Tables for Crystallography that is devoted to the sub- 
ject of 'Powder Diffraction' and will be published in 2014. ${ }^{3]}$

\section{Traditional Applications of X-ray Powder Diffraction}

The powder diffraction pattern of a crystalline phase is unique, so it can be used as a fingerprint to identify any known phases in a new material. Indeed qualitative phase identification and quantitative phase analysis are probably the main applications of powder diffraction in laboratories around the world. But a powder diffraction pattern contains much more information (Fig. 2).

The position of a peak $\left(\right.$ in $^{\circ} 2 \theta$ ) in a conventional powder diffraction pattern, for example, is determined by the $d$-spacing of the lattice planes giving rise to the reflection hkl via Bragg's law:

$$
\lambda=2 d_{h k l} \sin \theta
$$

where $\lambda$ is the wavelength of the $\mathrm{X}$-radiation. This means that, in principle, by measuring the positions of a handful of peaks in the pattern, and assigning $h k l$ indices to the associated $d$-spacings, the lattice parameters $a, b, c, \alpha, \beta$ and $\gamma$ can be derived. This procedure, known as indexing a pattern, is not without its pitfalls, but several different computer algorithms have been developed over the years and it is usually possible to assign the $h k l$ indices with at least one of them. ${ }^{[4]}$ Once the unit cell has been established, further information regarding the symmetry can be extracted by looking for classes of systematically absent reflections. For example, if all observed $h k 0$ reflections have even $k$ indices (i.e. all odd $k$ are absent), there is probably a $b$ glide plane perpendicular to the $c$ axis. In this way, the number of probable space groups can be narrowed down. It must be noted though, that identifying systematic absences in an XPD pattern can be difficult, because they are often obscured by reflection overlap. This means that the determination of the space group is often ambiguous, but usually some information about the symmetry can be learned.

The width of a peak in an XPD pattern is a result of several factors: the instrumental function of the diffractometer used, the crystallite size, and stress or strain within the crystallites. The instrumental contribution to the peak width with a laboratory diffractometer equipped with a conventional X-ray source tends to be larger ( $c a$. $0.05-0.10^{\circ} 2 \theta$ ) than that of a high-resolution instrument at a synchrotron facility (ca. $0.01-0.03^{\circ} 2 \theta$ ). This is why synchrotron experiments are preferred for complex structures. The sharper peaks cannot eliminate reflection overlap, but they do minimize it.

As the number of unit cells decreases below $c a .1000$ in any direction, the assumption of an infinite number of repeats becomes less applicable, and the peaks start to broaden. For example, if all $h 00$ reflections in an XPD pattern are broader than other reflections, it might be a result of the crystal morphology, if the crystal is particularly small along the [100] direction. On the other hand, the broadened peaks might be indicative of a spread of $d$-spacings along the [100] direction resulting from an externally applied pressure. Stress and strain cause small differences in the dimensions of the individual unit cells within the crystal, and this in turn affects the range of $d$-spacings (and therefore $2 \theta$ angles) observed along specific directions.

In a first approximation, if the stress/ strain is known to be negligible, the Scherrer equation, which was derived by Paul Scherrer in 1918 in the very early days of XPD and is still used today, ${ }^{[5]}$ can be applied to estimate the crystallite size:

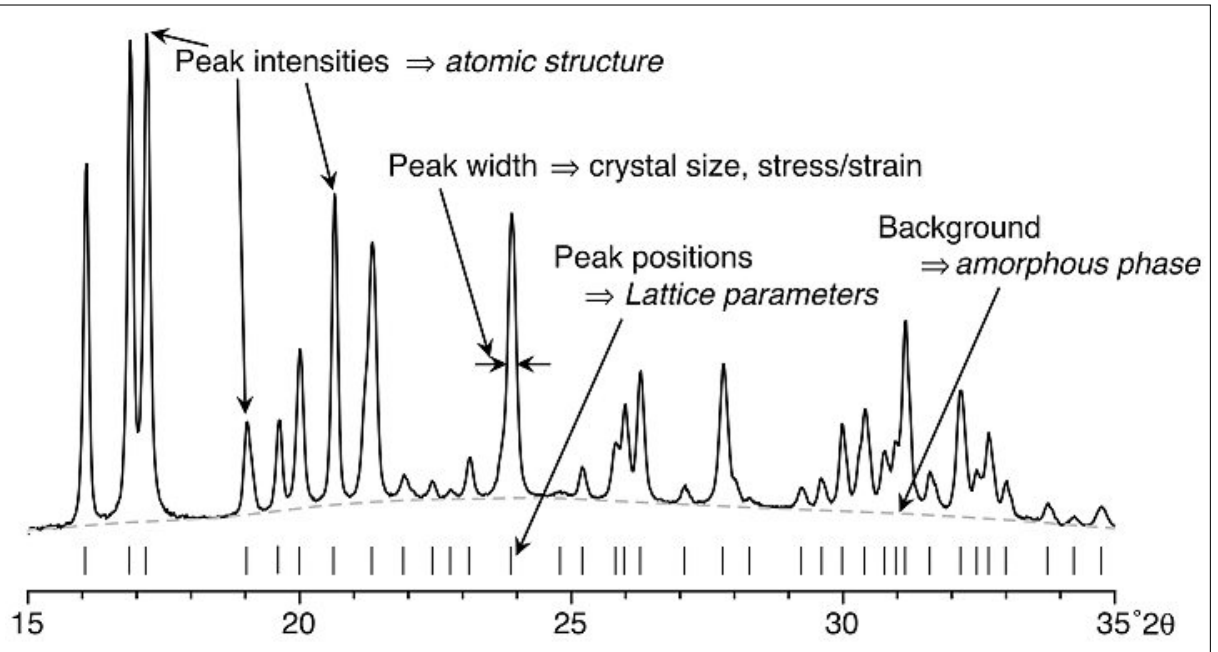

Fig. 2. The features of a powder diffraction pattern and the information they contain.

$$
\tau=\frac{K \lambda}{\beta \cos \theta}
$$

where $\tau$ is the mean size of the crystalline domains, $K$ is a shape factor with a value of $c a .0 .9, \lambda$ is the $\mathrm{X}$-ray wavelength, $\beta$ is the full peak width at half maximum minus the instrumental contribution, and $\theta$ is the diffraction angle. An analysis of the whole diffraction profile, on the other hand, can yield even more detailed information about crystallite size, shape, stress, strain, defects, etc. ${ }^{[6]}$

The background in an XPD pattern contains information about amorphous material in the sample, and unindexed peaks can often be used to identify one or more impurity phases.

\section{Structure Analysis Using Powder Diffraction Data}

For structure analysis, of course, it is the intensities of the reflections $\left(I_{h k l}\right)$ that contain the information we seek. For readers who are not very familiar with crystallography, the basic equations for structure analysis using X-ray diffraction data are presented briefly below. In general, the intensities are proportional to the square of the corresponding structure factor $F_{h k l}$ :

$$
I_{h k l} \propto F_{h k l}^{2}
$$

As the name suggests, these structure factors contain the information about the arrangement of the atoms within the unit cell:

$$
\begin{aligned}
F_{h k l}= & \sum_{n} f_{n}\left(\cos \left(2 \pi\left(h x_{n}+k y_{n}+l z_{n}\right)\right)\right. \\
& \left.+i \sin \left(2 \pi\left(h x_{n}+k y_{n}+l z_{n}\right)\right)\right)
\end{aligned}
$$

where $f_{n}$ is the scattering factor for atom $n$ and $x_{\mathrm{n}} y_{\mathrm{n}} z_{\mathrm{n}}$ are its fractional coordinates within the unit cell. For X-rays, the scattering factors reflect the number of electrons in the atom and are thus a measure of how strongly the X-ray beam is scattered by the respective atom. To emphasize that $F_{h k l}$ is a vector quantity, with an amplitude $\left|F_{h k l}\right|$ and a direction (phase angle $\phi_{h k l}$ ), this is sometimes written in the form:

$$
\begin{aligned}
F_{h k l} & =\sum f_{n} e^{2 \pi i\left(h x_{n}+k y_{n}+l z_{n}\right)} \\
& =\sum f_{n} e^{i \varphi_{n}}=\left|F_{h k l}\right| e^{i \varphi_{h k l}}
\end{aligned}
$$

For centrosymmetric structures, $\phi_{h k l}$ can only be 0 or $\pi$ (i.e. $\pm \mid F_{h k l}$, there is no 
imaginary component), but for non-centrosymmetric structures, $\phi_{h k l}$ can have any value between 0 and $2 \pi$.

Finally, the electron density at any point $X Y Z$ in the unit cell $\left(\rho_{X Y Z}\right)$ can be calculated by using these structure factors as coefficients in a Fourier series:

$$
\rho_{X Y Z}=\frac{1}{V_{u c}} \sum_{h k l}\left|F_{h k l}\right| \cos \left(2 \pi(h X+k Y+l Z)-\phi_{h k l}\right)(6)
$$

From Eqn. (6), it is clear that even with single-crystal data, there is a problem. Although the amplitude $\left|F_{h k l}\right|$ is measured, the phase angle $\phi_{h k l}$ is not. This is known as the phase problem, and solving a crystal structure from diffraction data is essentially equivalent to solving this phase problem.

Methods addressing the phase problem for single-crystal data started to appear as early as 1935, when Patterson introduced his $F^{2}$-series approach that we know today as the Patterson method. ${ }^{[7]}$ In short, a Patterson map $P_{u v w}$ is generated using $F^{2}$ as coefficients in the Fourier series instead of $F$ (thereby circumventing the phase problem):

$$
P_{u v w}=\sum_{h k l}\left|F_{h k l}\right|^{2} e^{-2 \pi i(h u+k v+l w)}
$$

and the result is a map showing the interatomic vectors between all atoms in the unit cell. All vectors begin at the origin, and the size of a peak in this map is proportional to the product of the scattering factors of the two atoms involved. That is, the most intense peaks will be between the heaviest scatterers (i.e. those with the most electrons). From such maps it is usually possible to deduce the positions of the heavier atoms in the structure. These atoms dominate the phases, so once their positions are known, the remaining atoms can then be found from an electron density map calculated using the phases calculated from the positions of the heavy atoms. Patterson maps for organic structures or for other materials containing elements with similar scattering powers (e.g. intermetallic compounds) are less straightforward to interpret, because no single set of peaks dominates the map.

In an attempt to address such 'equalatom' structures, Sayre derived the equation that now bears his name. ${ }^{[8]}$ The Sayre equation shows that the phases of the reflections $h k l, h^{\prime} k^{\prime} l$ ' and $h-h h^{\prime} k-k^{\prime} l-l$ ', for example, are related to one another (ultimately via the electron density), if all three are strong. The only assumptions are that the atoms in the structure are well-resolved in space (atomicity) and that the electron density is non-negative everywhere (positivity). Only centrosymmetric structures were considered. The Sayre equation is the basis for direct methods of structure solution, which has blossomed into the most powerful structure determination algorithm at our disposal today. Many years would pass, however, before either the Patterson or direct methods could be applied to XPD data.

It is interesting to note, that even in the very first powder diffraction experiments performed by Debye and Scherrer in Göttingen, Germany almost 100 years ago, ${ }^{[9]}$ it was recognized that a unit cell could be derived from the positions of the peaks and that the intensities of these peaks should allow the periodic arrangement of the atoms in the structure to be deduced. Indeed they derived the structures of $\mathrm{LiF}$ and $\mathrm{Si}$ from their powder diffraction patterns in 1916. In those early years, the structures of other simple cubic and hexagonal materials were also determined in a similar manner in a number of different laboratories.

It was not until the late 1940s, however, that the first lower symmetry crystal structures were determined using XPD data. In 1948, Zachariasen reported the structures of a series of $5 f$-element salts resulting from the Manhattan Project, ${ }^{[10]}$ and he used some ingenious methods to determine them. In particular, he examined the diffracted intensities for trends within different classes of reflections to estimate where the atoms were likely to be in the unit cell. It must be noted that this was done without the benefit of computers. Later, in 1963, he also exploited anisotropic thermal expansion, which affects the relative positions of the reflections and the pattern of their overlap as the unit cell changes with temperature, to obtain more information about the individual intensities of overlapping reflections and thereby solve the monoclinic structure of $\beta$-plutonium. ${ }^{[11]}$ This concept would be revisited by other powder diffractionists some 30 years later. ${ }^{[12]}$

In the $50 \mathrm{~s}$ and $60 \mathrm{~s}$, algorithms for direct methods and for calculating and interpreting Patterson maps developed rapidly, and crystallographers focused on the application of these methods to single-crystal data. Manual intervention was usually necessary, but the computer algorithms eased the way, and model building as a method of structure determination began to fall into disuse. With XPD data, however, there was no alternative.

\section{Structure Refinement Using Powder Diffraction Data}

Even if a plausible structure had been found by model building, either by con- structing a physical model or by using pencil and paper in conjunction with deductive reasoning, refining it with XPD data was problematic. Extracting reliable $\left|F_{h k l}\right|$ values for individual reflections from a powder pattern to use in the least-squares minimization procedure was not straightforward. With his seminal paper describing a whole-profile approach to crystal structure refinement using neutron powder diffraction data in 1969, Rietveld provided a solution to this problem. ${ }^{[13]}$ His algorithm challenged the computing power available at the time, but it allowed crystallographers to circumvent the problem of overlapping reflections. The diffraction pattern for the whole $2 \theta$ range was calculated for the structural model and compared point by point with the measured one, so observed peaks did not have to be deconvoluted into individual reflections. The original algorithm was written for constant-wavelength neutron data, whose peaks could be described very well with rather broad Gaussian functions. Attempts to adapt the method to XPD data were started almost immediately, but it proved to be quite a complicated undertaking. First, it was difficult to find a suitable peak shape function for X-ray experiments, ${ }^{[14]}$ and second, the sharpness of the peaks dictated that a much finer sampling of the $2 \theta$ values be performed. Nonetheless, by the mid 1980s, several programs that worked with XPD data had been written and applied successfully.

In the same period, powder diffractometers at synchrotron facilities were becoming accessible, so diffraction patterns with much sharper diffraction maxima could be measured.[15] It was soon established that surprisingly complex structures could be refined when such high-quality data were available. Of course, the significant increase in computing power also played an important role here. It was almost inevitable then that researchers would begin to explore the possibility of using such high-quality XPD data not only to refine structures, but also to solve them. Could high-resolution XPD data be used in the same way as single-crystal data to solve the phase problem directly, despite the overlap problem?

\section{Inorganic Structures Containing Heavy Atoms}

The first attempts to solve crystal structures directly from powder diffraction data instead of via model building were concentrated on highly crystalline inorganic materials containing heavy atoms. In 1977, Berg and Werner demonstrated that the Patterson method could be applied to XPD data. ${ }^{[16]}$ The key to their success 
was their development of a densitometer to digitize films recorded with strictly monochromatic $\mathrm{CuK} \alpha$, radiation and a GuinierHägg camera, which produced extremely sharp diffraction lines. ${ }^{[17]}$ An interpretable Patterson map could be generated from such XPD data. Indeed, the Patterson approach proved to be relatively robust with respect to incorrectly estimated intensities, as long as there were enough correctly estimated ones (i.e. non-overlapping reflections). ${ }^{[18]}$

Many structures followed, including $\mathrm{CaCl}_{2}\left(\mathrm{NH}_{3}\right)_{8}, \quad \mathrm{CaCl}_{2}\left(\mathrm{NH}_{3}\right)_{2}$ and $\mathrm{CaClOH}$ solved by Westman et al., [19] $\mathrm{KCuNb}_{3} \mathrm{O}_{9}$ and $\mathrm{KCuTa}_{3} \mathrm{O}_{9}$ by Groult et al., ${ }^{[20]} \mathrm{ZrKH}\left(\mathrm{PO}_{4}\right)_{2}{ }^{[21]}$ and $\mathrm{ZrNaH}\left(\mathrm{PO}_{4}\right)_{2}{ }^{[22]}$ by Clearfield et al., $\alpha-\mathrm{CrPO}_{4}$ by Attfield et al., ${ }^{[23]}$ and $\mathrm{KCaPO}_{4}$ by Louër et al.[24] These are just a few selected examples of the types of structures that could be addressed by applying the Patterson function to powder diffraction data in the 1980s. These structures have between 3 and 16 atoms in the asymmetric unit.

The application of direct methods, on the other hand, was more problematic, because the approach is a statistical one that is based on knowing which reflections are strong and which are weak. Nolang and Tergenius were able to solve the structure of $\mathrm{MnP}_{4}$ from laboratory XPD data using direct methods in 1980, but they used poor single-crystal data to get better estimates of the relative intensities of overlapping reflections. ${ }^{[25]}$ It took another six years before the first successes of applying direct methods to powder diffraction data alone were reported. These included the structures of $\mathrm{FeAsO}_{4}$ from high-resolution neutron powder diffraction data, ${ }^{[26]} \mathrm{Al}_{2} \mathrm{Y}_{4} \mathrm{O}_{9}$, $\mathrm{I}_{2} \mathrm{O}_{4}{ }^{[27]}$ and the zeolite sigma-2[28] from synchrotron XPD data, and a zeolitelike aluminophosphate, ${ }^{[29]} \mathrm{LiSbWO}_{6},{ }^{[30]}$ $\mathrm{Ba}_{2} \mathrm{PdO}_{3}{ }^{[31]}$ and $\mathrm{Li}_{2} \mathrm{TbF}_{6}{ }^{[32]}$ from laboratory XPD data. In a study of $\mathrm{LaMo}_{5} \mathrm{O}_{8}$, the overlapping reflections in high-resolution synchrotron XPD data were partitioned using electron diffraction data before direct methods were applied.[33] This idea was also used much later in the solution of particularly complex structures. In most of these examples, direct methods were used to locate the heavier scatterers, and then difference electron density maps and/ or chemical considerations were applied to complete the model.

\section{Zeolites}

Zeolites are framework aluminosilicates containing channels and cavities that are filled with exchangeable cations and removable water molecules. They are usually synthesized in polycrystalline form, so their structures, which are the basis of their commercially important ion-exchange, adsorption and catalytic properties, cannot be solved by conventional single-crystal methods. These materials rarely contain what we would call 'heavy' atoms, but they are generally more crystalline (i.e. the long range order is better) and adopt a higher symmetry than their organic counterparts, so they were ideal test cases for crystallographers developing methods of structure determination from XPD data.

By 1995, 105 zeolite framework types had been identified and assigned a 3-letter code by the International Zeolite Association. ${ }^{[34]}$ Of these, 48 had been determined from XPD data, 43 by model building and 5 by direct methods. Most of the other 57 were natural zeolites, for which single crystals could be found. Even some of those structures had to be solved by model building. In other words, there was a rich legacy of model building experience in the zeolite community. A few examples include the first synthetic zeolite (zeolite A) in $1956,{ }^{[35]} \mathrm{ZK}-5$ in $1965,{ }^{[36]}$ zeolite L in $1969^{[37]}$ and zeolite rho in 1973. ${ }^{[38]}$ Even today, this approach has its advantages in the field of zeolites. The structures of IPC$4,{ }^{[39]} \mathrm{COK}-14[40]$ and SSZ-52,[41] for example, were all solved manually by model building (albeit using computer graphics tools that were not available 20 years ago) within the last year.

In 1989, shortly after the first successful applications of direct methods to zeolites, ${ }^{[28,29]}$ a new approach specific to zeolites was reported. Deem and Newsam had devised an algorithm to build zeolite framework structures automatically, given a unit cell, its symmetry and the number of tetrahedral nodes ( $\mathrm{T}$ atoms such as $\mathrm{Si}, \mathrm{Al}$ or $\mathrm{P}$ ) per unit cell, by applying a simulating-annealing global-optimization algorithm. ${ }^{[42]}$ They used pseudo-potentials calculated on the basis of known zeolite structures to describe optimal T-T distances and T-T-T angles and these potentials were used to evaluate computer-generated arrangements of T-atoms in the unit cell. The algorithm was applied successfully to an unknown zeolite structure for the first time in 1996[43] and was updated to include parallel tempering in 1999. [44]

Another zeolite-specific algorithm, Focus, was reported in 1996.[45] It was first applied to the relatively complex structure of the zincosilicate VPI-9[46] and has since been used to solve 17 more novel framework structures. ${ }^{[47]}$ In contrast to the simulated annealing approach, which only uses the diffraction pattern passively to check the correctness of the models generated, Focus uses a dual-space approach. Random phases are assigned to the intensity data (in what crystallographers call reciprocal space) to generate a random electron density map (in real space). Overlapping reflections are usually simply equipartitioned. Chemical information about zeolite structures is then used to interpret those maps. From this model, a new set of phases is calculated and applied to the observed intensities to generate a new map. This Fourier recycling loop is then repeated until the phases converge or the maximum number of cycles prescribed is reached. Then the whole process is repeated with a new set of random starting phases. The inclusion of zeolite-specific information in the interpretation step (i.e. a 3-dimensional, 4-connected network of T atoms is expected) appears to compensate, at least in part, for the ambiguous diffraction intensities.

\section{Organic Compounds}

With a certain amount of effort, most organic and organometallic compounds can be persuaded to crystallize in the form of single crystals. Those that resist, however, do tend to form polycrystalline materials. Many pharmaceuticals fall into this category. In general, the XPD patterns of such materials do not extend as far in $2 \theta$ as their inorganic counterparts, but they often contain sufficient information for a reliable structure refinement, assuming that a starting model can be found. The problem is in getting that starting model. Single-crystal direct methods programs were found to work for simpler centrosymmetric structures containing one or two heavy atoms such as $\mathrm{S}$ or a metal. However, the quality of the XPD pattern is often insufficient for direct methods. In particular, there tend to be too few reflections with small $d$-spacings, which are essential for direct methods. Tremayne et al. applied the concepts of maximum entropy to the problem with some success, ${ }^{48]}$ but considerable manual intervention was required. In the early to mid 90s, the groups of Schenk, ${ }^{[49]}$ Giacovazzo ${ }^{[50]}$ and Rius ${ }^{[51]}$ began adapting their single-crystal direct methods programs to address the problems posed by powder diffraction data, and as a result, structure solution from XPD data using direct methods improved substantially. The programs EXPO ${ }^{[52]}$ and XLENS ${ }^{[53]}$ represent the current state of that art.

As the efforts to modify direct methods programs were beginning, several other groups began exploring the possibility of tailoring computer-assisted model-building algorithms to molecular structures. The reasoning was that a real-space approach would circumvent both the overlap problem and the lack of data at higher $2 \theta$ angles. The difference between molecular and zeolite structures in this context is that the connectivity of the atoms in a molecule is generally known from other character- 
ization techniques. It is the conformation/ configuration and packing in the unit cell of the crystal that needs to be determined. This means that the structure solution problem can be simplified by using internal coordinates (bond distances, bond angles and torsion angles) to describe the molecule. Only the position and orientation of the molecule in the unit cell and the free internal parameters (e.g. some torsion angles) need to be modified during the course of the global optimization procedure. In this way the number of parameters can be reduced significantly and the computing time kept within reason. Within two years of one another, Monte Carlo methods were implemented by Kariuki et al. ${ }^{[54]}$ and Andreev et al. ${ }^{[55]}$ simulated annealing by David et al.[56] and Andreev and Bruce, ${ }^{[57]}$ and genetic algorithms by Kariuki et al. ${ }^{[58]}$ and David et al. ${ }^{[59]}$ The viability of these approaches, in particular simulated annealing, can be seen very clearly in the explosion of structure determinations of molecular structures from powder diffraction data since the beginning of this century (Fig. 3). Today the most popular real-space programs are probably $F O X .^{[61]}$ Topas $^{[62]}$ and DASH. ${ }^{[63]} \mathrm{A}$ recent addition to this group of real-space approaches is the RAMM algorithm within the program $E X P O$, which takes advantage of the electron density map interpretation tools that have been developed for direct methods in that program. ${ }^{[64]}$

\section{Charge Flipping}

In 2004, Oszlanyi and Suto introduced a new method of structure solution for single-crystal data that they called charge flipping (CF). ${ }^{[65]}$ Like Focus, CF is a dual-space approach to the phase problem. The algorithm starts much like Focus, by assigning random phases to the reflections to generate an electron density map. Reasoning that negative electron density is not possible, the values for all pixels in the map with a density below a threshold value $\delta$ (small positive number) are multiplied by -1 (i.e. the sign is 'flipped') to produce a perturbed electron density map. Fourier transform of this map produces a set of calculated amplitudes $\left|F_{h k l}\right|$ with phases $\phi_{h k l}$. These phases are applied to the measured amplitudes to produce a new electron density map and the cycle is repeated. When the calculated and measured amplitudes match, the structure is solved.

Within a very short period of time, this algorithm was adapted to work with XPD data by Wu et al. in Arizona ${ }^{[66]}$ and Baerlocher et al. in Switzerland. ${ }^{[67]}$ In both implementations, a repartitioning of the overlapping reflections during the procedure was introduced, but this was coupled

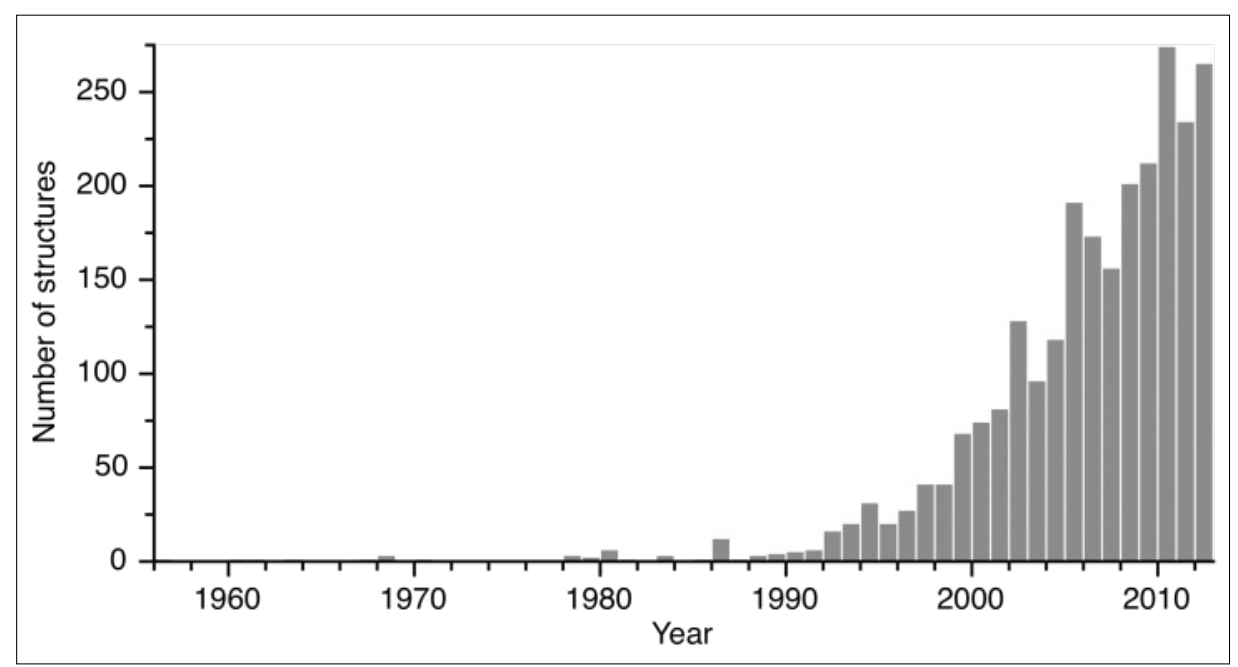

Fig. 3. The number of molecular structures solved from powder diffraction data from the time of the first structure in 1956 to the present (data extracted from the Cambridge Structural Database ${ }^{[60]}$.

to a second perturbation in the form of histogram matching[68] in the latter. In the short time since, the powder charge flipping $(p C F)$ algorithm has been applied successfully to a wide variety of problems, including zeolites, ${ }^{[69]}$ minerals, ${ }^{[70]}$ metal organic frameworks, ${ }^{[71]}$ organometallics ${ }^{[72]}$ and organic compounds. ${ }^{[73]}$ The two most readily accessible programs for powder charge flipping are Superflip ${ }^{[74]}$ and Topas. ${ }^{[62]}$

Combinations of charge flipping with other methods such as electron microscopy, ${ }^{[75]}$ IR spectroscopy, ${ }^{[76]}$ simulated annealing ${ }^{[77]}$ and maximum entropy ${ }^{[78]}$ have allowed the limits of complexity that can be addressed with powder diffraction data to be extended even further. Indeed, by using the $p C F$ algorithm in Superflip to combine high-resolution XPD data with phase information derived from just a few HRTEM images, the structures of two of the most complex zeolites known, IM-5 $(24 \mathrm{Si}+47 \mathrm{O} \text { in the asymmetric unit })^{[79]}$ and SSZ-74 $(23 \mathrm{Si}+48 \mathrm{O}+16 \mathrm{C}+2 \mathrm{~N}$ in the asymmetric unit) ${ }^{[80]}$ could be determined.

\section{Microcrystal Diffraction}

As synchrotron sources have become more intense and detectors more sensitive, the size of crystal required to collect highquality single-crystal diffraction data has decreased to just a few microns. Recently, data collected on a $2 \times 2 \times 8 \mu^{3}$ crystal of the zeolite SSZ-57 with a Pilatus $6 \mathrm{M}$ pixel detector ${ }^{[81]}$ at the Swiss Light Source (SLS), for example, was of such high quality that not only could its modulated structure (8-fold along one direction to give a $c$ axis of $110 \AA$ ) be determined, but the disorder in the structure could also be interpreted in quite some detail from the 3D diffuse scattering. ${ }^{[82]}$ Indeed, the boundary between single microcrystal and powder diffraction is becoming less distinct.

With electron diffraction, of course, crystals in the range of even $\sim 50 \mathrm{~nm}$ can be studied, but those reflection intensities are somewhat distorted by multiple and dynamical scattering effects, and this makes them more difficult to interpret. However, electron microscopy techniques are presently developing at a rapid pace and electron crystallography is slowly transforming from a technique for experts only to one that can also be used sensibly by nonspecialists. ${ }^{[83]}$

As the crystals that can be studied with $\mathrm{X}$-rays get smaller, it becomes increasingly difficult to rotate them in a controlled manner within the correspondingly small synchrotron beam. The white-beam Laue diffraction approach, in which a crystal is irradiated with multiple wavelengths simultaneously, offers an alternative to the traditional monochromatic experiment. With a white beam, a considerable portion of the diffraction data can be recorded with a single exposure without rotating the sample or moving the $2 \mathrm{D}$ detector. This is why it is often used for in situ experiments. The Laue method was developed quite extensively by the protein community, albeit for larger crystals, in the 1980s, but then other methods began to dominate that field and the white-beam approach was left more or less dormant for the following 25 years. Adaptations of the method for application to micron or perhaps even sub-micron sized non-protein crystals show that data can be collected on such crystals, and that these data are of a quality sufficient for solving and refining a structure. ${ }^{[84]}$ In other words, single-crystal data can be obtained from submicron-sized inorganic crystals. This does not signal the end of structure 
analysis using powder diffraction data, because the Laue microdiffraction setup is not readily available and there are still many polycrystalline materials that are not amenable to the method, but it does offer some exciting possibilities.

In 2016, the Swiss X-ray Free Electron Laser facility, SwissFEL, at the Paul Scherrer Institute (PSI) in Villigen, Switzerland is scheduled to open its doors to users. This new type of $\mathrm{X}$-ray source will produce very short bursts (1-60 femtoseconds) of extremely intense X-rays with laser-like properties. One of the unique features of SwissFEL that sets it apart from the other four X-FEL projects around the world is that its specifications include a $4 \%$ bandpass operating mode. ${ }^{[85]}$ That is, the beam must not be strictly monochromatic. Simulations show that this feature can be used to advantage in single-shot diffraction experiments on very small non-protein crystals. ${ }^{[86]}$ Only single shots are possible, because the intensity of the beam destroys the sample, but not before a diffraction pattern is generated. Although a $4 \%$ bandpass is a long way from the white beam used in Laue diffraction experiments, many of the ideas developed for the Laue experimental setup and data analysis can be transferred to the SwissFEL experiment.

\section{Powder Diffraction in Switzerland}

Swiss scientists have been involved in $\mathrm{X}$-ray powder diffraction from the very beginning, when the Swiss student Paul Scherrer, with his supervisor Peter Debye, devised the Debye-Scherrer camera for powder diffraction experiments back in 1916. [9] That geometry is still one of the predominant experimental setups for powder diffraction experiments today, especially at synchrotron radiation facilities. Structure analysis using powder diffraction data has played a key role in a number of long-standing research programs at many Swiss universities over the last 50 years. It has been used, for example, to study zeolites and magnetic structures at the ETH in Zurich, metal hydrides in Geneva, minerals in Bern, and molecular structures in Neuchatel and Basel. This interest has led quite naturally to the development of methodology, in particular in Geneva, Lausanne and Zurich, and to the development of the Mythen detector ${ }^{[87]}$ at PSI. The importance of powder diffraction to Swiss research is reflected not only in the large number of publications in the area, but also in the support that the powder diffractometers continue to enjoy at the neutron source SINQ and the synchrotron source SLS (both at the PSI) and on the Swiss-Norwegian Beamlines (SNBL) at the ESRF in Grenoble. With the current state of the art of powder diffraction and SwissFEL on the horizon, scientists in Switzerland with polycrystalline materials can look forward to a bright future.

\section{Received: October 29, 2013}

[1] 'Structure Determination from Powder Diffraction Data', Eds. W. I. F. David, K. Shankland, L. B. McCusker, Ch. Baerlocher, Oxford University Press, Oxford, 2002.

[2] Z. Kristallogr. 2004, 219, 782-901.

[3] 'International Tables for Crystallography, Volume H: Powder Diffraction', Wiley, 2014, in press.

[4] J. Bergmann, A. Le Bail, R. Shirley, V. Zlokazov, Z. Kristallogr. 2004, 219, 783

[5] P. Scherrer, Nachr. Ges. Wiss. Göttingen, MathPhys. Kl. 1918, 98.

[6] P. Scardi, M. Ortolani, M. Leoni, Mater. Sci. Forum 2010, 651, 155.

[7] A. L. Patterson, Z. Kristallogr. 1935, 90, 517.

[8] D. Sayre, Acta Crystallogr. 1952, 5, 60.

[9] P. Debye, P. Scherrer, Nachr. Ges. Wiss. Göttingen, Math-Phys. Kl. 1916, 1.

[10] W. H. Zachariason, Acta Crystallogr. 1948, 1, 265.

[11] W. H. Zachariason, Acta Crystallogr. 1963, 16, 369.

[12] P. Fernandes, K. Shankland, W. I. F. David, A. J. Markvardsen, A. J. Florence, N. Shankland, C. K. Leech, J. Appl. Crystallogr. 2008, 41, 1089.

[13] a) H. M. Rietveld, J. Appl. Crystallogr. 1969, 2, 65; b) 'The Rietveld Method', Ed. R. A. Young, Oxford University Press, Oxford, 1993.

[14] P. Suortti, in 'The Rietveld Method', Ed. R. A. Young, Oxford University Press, Oxford, 1993, pp. $167-185$.

[15] J. B. Hastings, W. Thomlinson, D. E. Cox, J. Appl. Crystallogr. 1984, 17, 85.

[16] J.-E. Berg, P.-E. Werner, Z. Kristallogr. 1977, $145,310$.

[17] P.-E. Werner, T. Ekström, Anal. Chem. 1980, $52,833$.

[18] C. C. Wilson, Acta Crystallogr. 1989, A45, 833.

[19] S. Westman, P.-E. Werner, T. Schuler, W. Raldow, Acta Chem. Scand. 1981, A35, 467.

[20] D. Groult, M. Hervieu, B. Raveau, J. Solid State Chem. 1984, 53, 184

[21] A. Clearfield, L. B. McCusker, P. R. Rudolf, Inorg. Chem. 1984, 23, 4679.

[22] P. Rudolf, A. Clearfield, Acta Crystallogr. 1985, B41, 418.

[23] J. P. Attfield, A. W. Sleight, A. K. Cheetham, Nature 1986, 322, 620

[24] M. Louër, J. Plevert, D. Louër, Acta Crystallogr. 1988, $B 44,463$.

[25] B. I. Nolang, L.-E. Tergenius, Acta Chem. Scand. 1980, A34, 311.

[26] A. K. Cheetham, W. I. F. David, M. M. Eddy, R. J. B. Jakeman, M. W. Johnson, C. C. Torardi, Nature 1986, 320, 46 .

[27] M. S. Lehmann, A. Norlund Christensen, H. Fjelvag, R. Feidenhans'l, M. Nielsen, J. Appl. Crystallogr. 1987, 20, 123.

[28] L. B. McCusker, J. Appl. Crystallogr. 1988, 21, 305

[29] P. R. Rudolf, C. Saldarriaga-Molina, A. Clearfield, J. Phys. Chem. 1986, 90, 6122.

[30] A. Le Bail, H. Duroy, J. L. Fourquet, Mat. Res. Bull. 1988, 23, 447

[31] Y. Laligant, A. Le Bail, G. Ferey, M. Hervieu, B. Raveau, A. Wilkinson, A. K. Cheetham, Eur. J. Solid State Inorg. Chem. 1988, 25, 237.

[32] Y. Laligant, A. Le Bail, G. Ferey, D. Avignant, J. C. Cousseins, Eur. J. Solid State Inorg. Chem. 1988, $25,551$.
[33] S. J. Hibble, A. K. Cheetham, A. R. L. Bogle, H. R. Wakerley, D. E. Cox, J. Am. Chem. Soc. 1988, 110,3295 .

[34] a) C. Baerlocher, L. B. McCusker, D. H. Olson, 'The Atlas of Zeolite Framework Types', Elsevier, Amsterdam, 2007; b) C. Baerlocher, L. B. McCusker, http://www.iza-online.org/ databases/.

[35] D. W. Breck, W. G. Eversole, R. M. Milton, T. B. Reed, T. L. Thomas, J. Am. Chem. Soc. 1956, 78, 5963.

[36] W. M. Meier, G. T. Kokotailo, Z. Kristallogr. $\mathbf{1 9 6 5}, 121,211$.

[37] R. M. Barrer, H. Villiger, Z. Kristallogr. 1969, $128,352$.

[38] H. E. Robson, D. P. Shoemaker, R. A. Oglivie, P. C. Manor, Adv. Chem. Ser. 1973, 121, 106

[39] W. J. Roth, P. Nachtigall, R. E. Morris, P. S. Wheatley, V. R. Seymour, S. E. Ashbrook, P. Chlubna, L. Grajciar, M. Poozij, A. Zukal, O. Shvests, J Cejka, Nature Chem. 2013, 5, 628.

[40] E. Verheyen, L. Joos, K. Van Havenbergh, E. Breynaert, N. Kasian, E. Gobechiya, K. Houthoofd, C. Martineau, M. Hinterstein, F. Taulelle, V. Van Speybroeck, M. Waroquier, S. Bals, G. Van Tendeloo, C. E. A. Kirschhock, J. A. Martens, Nature Mater. 2012, 11, 1059.

[41] D. Xie, L. B. McCusker, C. Baerlocher, S.I. Zones, W. Wan, X. D. Zou, J. Am. Chem. Soc. 2013, $135,10519$.

[42] M. W. Deem, J. M. Newsam, Nature 1989, 342, 260.

[43] D. E. Akporiaye, H. Fjellvag, E. N. Halvorsen, J. Hustvelt, A. Karlsson, K.-P. Lillerud, J. Phys. Chem. 1996, 100, 16641.

[44] M. Falcioni, M. W. Deem, J. Chem. Phys. 1999, 110, 1754.

[45] R. W. Grosse-Kunstleve, L. B. McCusker, C. Baerlocher, J. Appl. Crystallogr. 1997, 30, 985.

[46] L. B. McCusker, R. W. Grosse-Kunstleve, C. Baerlocher, M. Yoshikawa, M. E. Davis, Microporou Mater. 1996, 6, 295.

[47] L. B. McCusker, C. Baerlocher in 'International Tables for Crystallography, Volume H: Powder Diffraction', Wiley, 2014, in press.

[48] M. Tremayne, P. Lightfoot, C. Glidewell, K. D. M. Harris, K. Shankland, C. J. Gilmore, G. Bricogne, P. G. Bruce, J. Mater. Chem. 1992, 2 , 1301.

[49] J. Jansen, R. Peschar, H. Schenk, Z. Kristallogr. 1993, 206, 33.

[50] G. Cascarano, L. Favia, C. Giacovazzo, J. Appl. Crystallogr. 1992, 25, 310.

[51] a) J. Rius, C. Miravitlles, J. Appl. Crystallogr. 1988, 21, 224; b) J. Rius, J. Sane, C. Miravitlles, J. M. Amigo, M. M. Reventos, D. Louer, Anal. Quimica 1996, 92, 223.

[52] A. Altomare, C. Cuocci, C. Giacovazzo, A. Moliterni, R. Rizzi, N. Corriero, A. Falcicchio, J. Appl. Crystallogr. 2013, 46, 1231.

[53] J. Rius, Acta Crystallogr. 2011, A67, 63.

[54] B. M. Kariuki, D. M. S. Zin, M. Tremayne, K. D. M. Harris, Chem. Mater. 1996, 8, 565.

[55] Y. G. Andreev, P. Lightfoot, P. G. Bruce, J. Appl. Crystallogr. 1997, 30, 294.

[56] W. I. F. David, K. Shankland, N. Shankland, Chem. Commun. 1998, 931.

[57] Y. G. Andreev, P. G. Bruce, J. Chem. Soc., Dalton Trans. 1988, 4071.

[58] B. M. Kariuki, H. Serrano Gonzalez, R. L. Johnston, K. D. M. Harris, Chem. Phys. Let 1997, 280, 189.

[59] K. Shankland, W. I. F. David, T. Csoka, Z. Kristallogr. 1997, 212, 550.

[60] F. H. Allen, Acta Crystallogr. 2002, B58, 380.

[61] a) V. Favre-Nicolin, R. Cerny, J. Appl. Crystallogr. 2002, 35, 734; (b) http://vincefn. net/Fox/

[62] a) A. Coelho, J. Appl. Crystallogr. 2000, 33, 899; b) http://www.topas-academic.net/

[63] a) W. I. F. David, K. Shankland, J. van de Streek, E. Pidcock, W. D. S. Motherwell, J. C. Cole, J. 
Appl. Crystallogr. 2006, 39, 910; b) http://www. ccdc.cam.ac.uk/Solutions/PowderDiffraction/

[64] A. Altomare, C. Cuocci, A. Moliterni, R. Rizzi, J. Appl. Crystallogr. 2013, 46, 476.

[65] G. Oszlányi, A. Sütô, Acta Crystallogr. 2004, A60, 134.

[66] J. Wu, K. Leinenweber, J. C. H. Spence, M. O'Keeffe, Nature Mater. 2006, 5, 647.

[67] C. Baerlocher, L. B. McCusker, L. Palatinus, Z. Kristallogr. 2007, 222, 47.

[68] K. Y. J. Zhang, P. Main, Acta Crystallogr. 1990, A46, 41.

[69] C. Baerlocher, F. Gramm, L. Massuger, L. B. McCusker, Z. B. He, S. Hovmoller, X. D. Zou, Science 2007, 315, 1113.

[70] H. W. Ma, D. L. Bish, H. W. Wang, S. J. Chipera, Am. Mineral. 2009, 94, 622.

[71] F. Gandara, F. J. Uribe-Romo, D. K. Britt, H. Furukawa, L. Lei, R. Cheng, X. F. Duan, M O'Keeffe, O. M. Yaghi, Chem. Eur. J. 2012, 18, 10595.

[72] J. A. Kaduk, M. A. Toft, J. T. Golab, Powder Diffraction 2010, 25, 19.
[73] D. Sisak, L. B. McCusker, A. Buckl, G. Wuitschik, Y. L. Wu, W. B. Schweizer, J. D. Dunitz, Chem. Eur. J. 2010, 16, 7224.

[74] L. Palatinus, G. Chapuis, J. Appl. Crystallogr. 2007, 40, 786.

[75] L. B. McCusker, C. Baerlocher, Z. Kristallogr. 2013, 228, 1.

[76] A. K. Inge, H. Fahlquist, T. Willhammar, Y. N. Huang, L. B. McCusker, X. D. Zou, J. Appl. Crystallogr. 2013, 46, 1094.

[77] A. K. Inge, S. L. Huang, H. Chen, F. Moraga, J. L. Sun, X. D. Zou, Cryst. Growth Des. 2012, 12, 4853.

[78] D. Sisak, C. Baerlocher, L. B. McCusker, C. J. Gilmore, J. Appl. Crystallogr. 2012, 45, 1125.

[79] C. Baerlocher, F. Gramm, L. Massuger, L. B. McCusker, Z. B. He, S. Hovmoller, X. D. Zou, Science 2007, 315, 1113.

[80] C. Baerlocher, D. Xie, L. B. McCusker, S. J. Hwang, I. Y. Chan, K. Ong, A. W. Burton, S. I Zones, Nature Mater. 2008, 7, 631.

[81] C. Broennimann, E. F. Eikenberry, B. Henrich, R. Horisberger, G. Huelsen, E. Pohl, B. Schmitt,
C. Schulze-Briese, M. Suzuki, T. Tomizaki, H. Toyokawa, A. Wagner, J. Synchrotron Rad. 2006, 13, 120.

[82] C. Baerlocher, T. Weber, L. B. McCusker, L. Palatinus, S. I. Zones, Science 2011, 333, 1134.

[83] X. Zou, S. Hovmöller, P. Oleynikov, 'Electron Crystallography', Oxford University Press, Oxford, 2011.

[84] C. Dejoie, L. B. McCusker, C. Baerlocher, M. Kunz, N. Tamura, J. Appl. Crystallogr. 2013, 46, doi:10.1107/S0021889813026307.

[85] B. D. Patterson, R. Abela, H. H. Braun, U. Flechsig, R. Ganter, Y. Kim, E. Kirk, E. Oppeit, M. Pedrozzi, S. Reiche, L. Rivkin, T. Schmidt, B. Schmitt, V. N. Strocov, S. Tsujino, A. F. Wrulich, New J. Phys. 2010, 12, 035012.

[86] C. Dejoie, L. B. McCusker, C. Baerlocher, R. Abela, B. D. Patterson, M. Kunz, N. Tamura, J. Appl. Crystallogr. 2013, 46, 791.

[87] A. Bergamaschi, A. Cervellino, R. Dinapoli, F. Gozzo, B. Henrich, I. Johnson, P. Kraft, A. Mozzanica, B. Schmitt, X. Shi, J. Synchr. Rad. 2010, 17, 653. 